\title{
Smart Kitchen - A Measurement System
}

\author{
M. A. M Azran \\ Faculty of Computing, \\ Sri Lanka Institute of \\ Information Technology \\ Colombo
}

\author{
M. F. M. Zaid \\ Faculty of Computing, \\ Sri Lanka Institute of \\ Information Technology \\ Colombo
}

\author{
M. S. M. Sakeeb \\ Faculty of Computing, \\ Sri Lanka Institute of \\ Information Technology \\ Colombo
}

\author{
M. M. M. Althaff \\ Faculty of Computing, Sri Lanka Institute of \\ Information Technology \\ Colombo
}

\author{
S. G. S. Fernando \\ Senior Lecturer, Faculty of Computing, Sri Lanka \\ Institute of Information Technology \\ Colombo
}

\begin{abstract}
The tasks in our lives are getting easier and easier with the rapid growth of technology. The world is moving into an era of smartness and everything is getting automated and is able to be controlled remotely using sensor technology. Kitchen is a vital area in a home where the activities in the future has space for improvement in the field of home automation. The ingredients of a kitchen needs to be monitored manually and decisions will have to be taken by the user. The research paper explains a system that can be used to measure kitchen ingredients in an automated manner. The sensors are being used to transfer data with the help of a Wi-Fi-shield which is used to connect the Arduino board and the data will be presented in the form of an android application to the user. This helps in budgeting income for a month and gives the user an idea how much will be needed for the next month. Included in the project is measuring gas level using a weight sensor and when the level drops the supplier is informed. Since the application is hosted on a server the user is able to view the data from anywhere as the data and the elements are connected using the internet of things concept.
\end{abstract}

\section{Keywords}

Sensor technology, home automation, wifi-shield, Arduino board, android application, budgeting income, weight sensor, internet of things

\section{INTRODUCTION}

The project is to automate certain processes done in a kitchen, nowadays it is a basic need for all people and everyone in the world is looking for a change in the way things are done. Sensor technology and internet of things are the basic concepts behind the project, this is where the entire kitchen is connected in a network and the elements are able to communicate the data between one and another. Currently everything is turning into automated processes and the management of the kitchen in a home is something very essential for the future [1].

Appropriate sensors are needed to measure the different elements and an Arduino board is used to connect the elements and to present the data. The data will be presented to the user in an android application format and the software should prove to be appealing to the user.
One of the most important aspects in a home is the management of the kitchen. Presently the kitchen is managed by a person and he/she has to take all decisions including: whether to fill a container which is finishing, what items are needed for the kitchen and also to monitor the gas level in the kitchen. In addition to this he has to check whether the income that is earned is enough to purchase the things needed. The use of sensors to measure the kitchen items and recording them in a database to present them in an android application to the users is the main aim behind the project that is planned to be implemented.

The programming of the Arduino board is used by the team to sense the items' level in the container and send the data to the

Database and it will be presented to the user as an android application.

The android application is one end of the system which is the users' end and will display the data in a creative and appealing fashion. The user will be able to view the number of products in the kitchen even if the user is outside the house and he will know what is needed to be bought for the kitchen.

The amount of gas in the cylinder in a kitchen will be able to be known through the android application by using load cell to measure the gas level. If the level is below a certain threshold value, the user will be notified through the application and the gas supplier will be contacted as well [2]

The objectives of this project is to:

1. To develop an application to detect the level of items in each container using weight sensors.

2. To detect the gas level and show a warning accurately.

3 . To connect the data from the kitchen and present it through an android application to the user.

4. To know how much of kitchen items are needed for the next month and calculate the amount needed for the next month.

\section{BACKGROUND STUDY}

Home automation is automation of the home. It commonly defines address that combine technology and services through home networking to advance the condition of living. Home automation is not a new term for science society and has been 
around for a significant time. Home automation is to modify condition, control of lighting, appliances, and other systems, to provide improved advancement, convenience, security and efficiency. For disabled and elderly person home automation can be the additional of institutional care [3].

Home automation has been increasing vastly in latest years due to much higher affordability and simplicity. Being able to control aspects of our houses, and for having the feature to respond automatically to events, it is becoming more and more popular and necessary due to security and cost purposes. S.Chattoraj proposes to implement an integrated home automation and security system. The project is low cost solution using off the shelf components to reduce cost and open source software to get around licensing requirements of software. An Arduino controls sensors and actuators that monitor a defined location and take action based on specified parameters like ambient light, temperature etc. The Arduino can also send messages if it detects an exception. The voice recognition conception allows the user to use voice commands to control his house [4]

Safety and security is most important for anything which we have in our daily life, especially in the home to prevent the explosion of gases. Now-a-days the explosion of domestic LPG is increasing, LPG scam is also increasing parallel with it. To avoid the frequently checking the gas physically and scam, the capacity of gas in cylinder is continuously monitored using a weight sensor load cell. The LPG Detection system presents how to detect the leakage using a gas sensor and book a new cylinder automatically by sending a message to the agency [5].

Home automation plans to use computer and information technology to control home appliances and features. Home networking is the core in the implementation of an automation system for a smart home. Hence every range prefers automated control systems most of the smart home applications are accepted with wireless networking methods, such as ZigBee, Wi-Fi, or radio frequency wireless communication. The project discusses a low cost, secure, ubiquitously accessible, auto-configurable, remotely controlled solution. The approach analysed here achieved the target to control home appliances remotely using the wireless technology to connect devices and satisfy user requirements. Wi-Fi technology has proved to provide remote control, home security and is cost effective as compared to the previously existing system [6].

R.Kamoda et.al aim to re-identify grocery when it is put into or taken from a refrigerator in order to automatically monitor grocery inventory. The system proposed using the load balance feature on a shelf in the refrigerator to reflect both the weight and the position of grocery items. The system can reidentify groceries using the load balance feature by means of a prototype device and the two simulated experiments. The experimental results show that the load balance feature allows fairly accurate grocery re-identification when grocery items are put on the load-sensing board in neat rows. It also shows that some grocery items could be incorrectly identified as others even with the load feature, when one of the grocery items having similar weight to another is stacked on it [7].

As a result of the busy life style of current generation, they avoid spending unnecessarily on items and services that do not contribute to achieving user budget. If users are working with limited resources, budget makes it easier to make ends meet. It clearly says that a result of new technology there is a new product always that appears to buy or be a test for people, so saving money is not easy. Some people think twice about their budget, but majority has failed [8].

From most of the budget exceeding items, unexpected food expenses are on the top most level. This statement show it clearly (this is a kind of survey), In day to day life we are unable to plan exact amount of food for the next week or month, but when the food wasted or spoils, our money is surely down the drain. Drawing a plan for our food items will help a lot, as does knowing the time our food keep fresh on the shelves or in the refrigerator. But at last all it takes is a little attention on saving on food items [9]

\section{METHODOLOGY}

\subsection{Planning}

Smart Kitchen - A Measurement system started off with reviewing the literature available about the concept that was behind the project, which is Internet of things and Home automation [10]. The project was derived from these concepts and the desire to make daily tasks done more easily. The reason that the kitchen area was chosen to be automated was because it is an area which is the heart of a home. If the processes done there can be successfully automated then the rest of the home can be focused in the future.

The ideas that were reviewed by the team were considerably large in size and functionalities to be implemented. The project had to be narrowed down to fit the team's potential and abilities. There were many areas which the team were not able to cover but in future will look to develop. It had to be narrowed down into four main components: the weight cell sensor and gas notification, the Wi-Fi shield module, the android application and finally the budget forecasting.

\subsection{Requirement Gathering and Analysis}

The project is based on different factors such as Technology, Controlling Finance, User Experience and User Preferences are the main factors that contribute to the Effective Management of a Kitchen. The proposed project collected data from a sample size of 52 individuals. The people were mainly housewives from which the data was collected. The sample was picked up from non-probability sampling method. This means that each person has no probability in being selected for the sample. The process was made simple and the method used to gather the data was questionnaires.

The questionnaires were distributed manually and online through the use of survey monkey. The sample which was easy to distribute were given hardcopies and certain sample were given online forms. In both ways data was collected, the size came to 52 respondents which were all housewives and working women. These women were around the age of 25 to 50 , which means it is a typical data set to be taken from. The sampling method used was a typical quota sampling which means a selected quota will be used to gather data. Also the project used snowball sampling in the case of the online forms which were made using survey monkey. The forms were forwarded to people but to the required sample only and the data can be assured to be of quality. The questions asked were particularly relevant to the topic of the project, which means they were effective. It included questions which had one option: yes or no, some questions had the users' agreement levels. They were basically statements like, I monitor my gas level, I always forget what to buy when comes to kitchen ingredients and I buy something that is already available at home. 
The data shown above was collected from housewives and working women. The reason is because it is women who mostly use a kitchen and the team had an idea that it would be best suitable for them to answer the questions. The data show that most of the people are actually concerned about their kitchen and would prefer making it smart.

The results show a positive impact on the project, most of them gave an opinion that was relevant and supportive to the project's problem.

\subsection{Design}

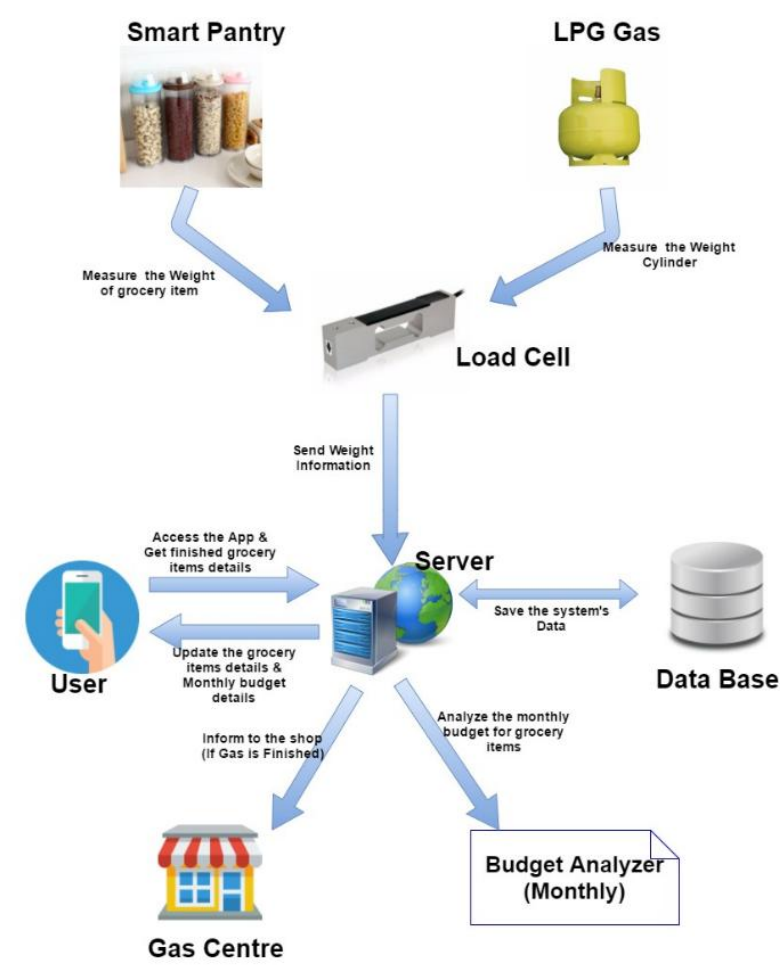

Figure 1 - System Architecture Diagram

The above shown figure shows how each component is linked with each other. The units to be measured, the ingredients and the gas level are measured using a load cell. This data is sent to a server that is hosted which contains the database. The budget component is linked to this and so is the gas center which will receive an order as per the gas level. The user will be able to view the data from an android application.

\subsection{Implementation}

The project uses the prototyping method to develop the system and testing will be done as well. Primarily, the hardest part of the project were done first and gradually moved onto the smaller parts of the project. The hardware components need to be purchased and checked whether they are working properly. Then move onto the testing the hardware and check for errors.

After the hardware has been checked the planning stage was completed and the project moved onto the design stage, this is where the software interfaces were designed along with the entire system design was done. The weight measurements were done using load cell sensors.

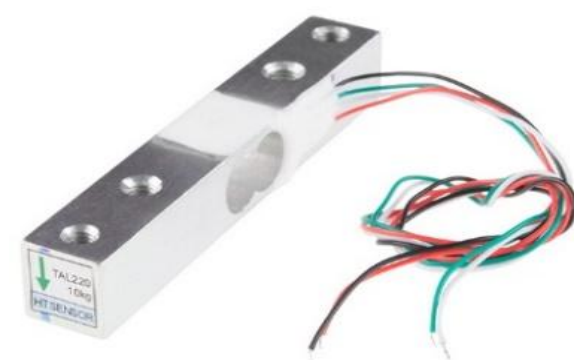

Figure 2 - Load cell

The implementation of the system was then done step by step following the order of importance and the coding will need to be done. The Arduino board will be coded to measure the weight from sensors of the kitchen items and the load cell to measure the gas level. The connection of the Arduino board to the android application can then be implemented.

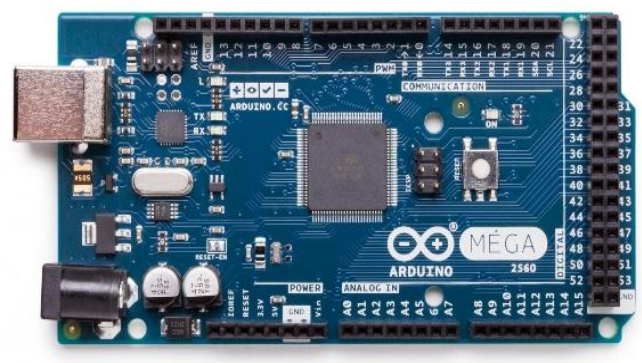

Figure 3 - Arduino Mega

Finally, the budgeting calculation was done to know how much the user will need to purchase the kitchen items that are needed for the next month. For this, certain budgeting algorithms were used.

Android programming platform was used to design the interfaces, android studio 2.3.1 was used to design them.

We conducted a survey to identify the current usage of these types of application in the society.

Survey results shows that most of the people are not using an ingredients weight measurement and budget management application.

Budget forecasting algorithm uses user item purchasing history, user preferences, and current pricing list. Budget is highly depends on users used item [8]. Budget forecasting helps users have more control in on their budget. The linear regression equation is shown below.

$$
\begin{aligned}
& Y=a+b x \\
& b=\frac{N_{\Sigma} X Y-\left(\Sigma^{X}\right)\left(\Sigma^{Y}\right)}{N_{\Sigma} X^{2}-\left(\Sigma^{X}\right)^{2}}, a=\frac{\sum^{Y-b_{\Sigma} X}}{N}
\end{aligned}
$$

Figure 4 - Budget Equation

$\mathrm{X}=$ Month's quantity

$\mathrm{Y}=$ Monthly Expense

$\mathrm{b}=$ the slope of the regression Line

$a=$ the intercept (on $y$-axis) 
$\mathrm{N}=$ number of pairs of data

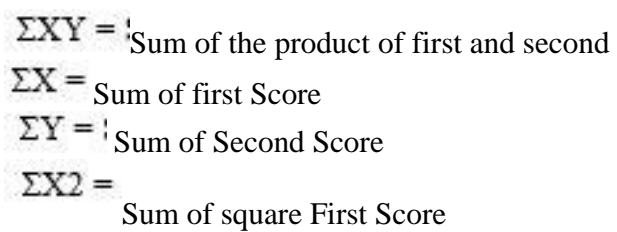

\subsection{Testing}

This section shows the results and their discussion that the research team achieved from the research project. The important implications of the research findings, regardless of the statistical significance of this research are discussed below. Further, Identifying the defect and limitation of this project can be useful for future researchers in order to continue their research. The major object of "Smart Kitchen A Measurement System" is to provide an efficient kitchen management system to the society.

\section{RESULTS AND DISCUSSION}

Smart Kitchen System comprises of three major components such as Load cells, Android application for users and budget system for the home users.

Primarily, the containers are designed to read and transmit the weight level details. This data with the gas level to be read and transmitted to the main server. Fig 5 below shows design and hardware implementation of the load cells.

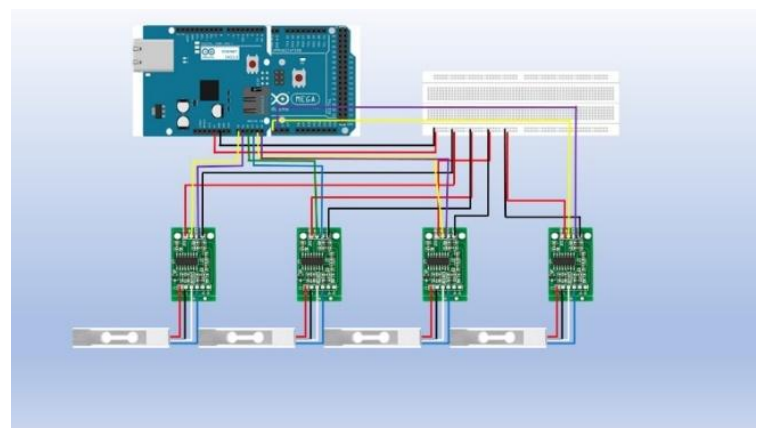

Figure 5 - Hardware Circuit Diagram

The data is then sent via a Wi-Fi module to the server in which the database is present. The android application in order retrieves the data and displays them in various forms as shown in the below figures.

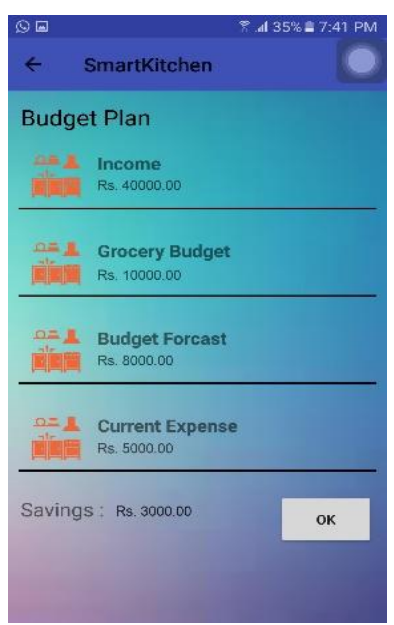

Figure 6 - Budget Information
The above figure shows the budget interface that user get when he gives his budget details. The user will be shown the savings he or she has finally.

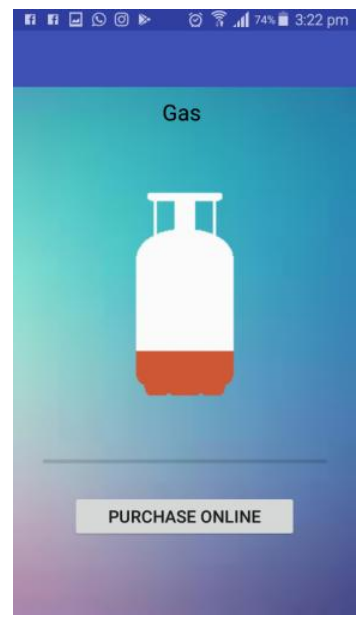

Figure 7 - Gas Interface

The above figure shows the gas level in the gas cylinder measured by the load cell, the user will be able to order the gas online.

Project team encountered numerous technical problems during the life time of product development. Major problem that project team encountered was managing software serial between two gadgets.

Calibrating load cell sensors to the max accuracy level and monitoring and replacing damaged circuit wires time to time were some other problems faced by the project team.

Load cell calibration was one of the tedious problem faced by the project team which took a huge amount of time during hardware assembling. Load cell sensors give random values as the output which differs with altitude even if weight of same object is measured. Initially project team was not aware of this issue as the team was hardly familiar with hardware programming.

Soon after this issue was found load cell sensor was kept at the same altitude as in the containers and calibrated. Project team found a rough equation for calibration by plotting known weights with load cell readings. Accuracy of reading were further refined by testing with minute changes. 


\section{CONCLUSION AND FUTURE WORK}

Rapidly increasing population, urbanization, and industrialization have made Kitchen management a global challenge. The Smart Kitchen system provided comprehensive solutions to the people those who are in the urban area. This system could read and transmit the weight data to the server. Finally, the project successfully achieved to fulfil all the objectives of this system and hope this research would be of benefit. The research team hopes that this study will be helpful for the researchers who are interested in the topics like budget management, smart kitchen systems as well as kitchen budgeting. It will also give better ideas and more knowledge to implement similar kind of projects with more advanced tools.

During the development of this project, the following are the limitations identified:

Wi-Fi module used to transfer data - as the data from the sensors is transferred using a Wi-Fi module the data is bound for security breaches.

Limited battery life - A 12v 8000mah li - ion battery has been used to power up Arduino circuit. It has a battery life of circular charge and discharge up to 500 times. Battery should be recharged in regular intervals for reliable functioning of the containers.

Requires a stable Internet Connection

Smart phone and basic knowledge is significant for domestic users.

As the data is saved in a server if the server is down the system will not work.

A smart kitchen management system covers a very large scope with an ultimate goal of zero waste in the system. Till the goal is met numerous number of research paths will be open to researchers. This research area will stay fresh with the rise of modern technologies. Project team has identified following as immediate set of future works which may interest researchers in this area:

Developing mobile application with multi-platform support.

Develop smart containers which have sensors fixed with them.

Create awareness of the IOT concept.

Expanding the system targeting kitchen management of entire nation.

\section{ACKNOWLEDGEMENT}

The project team of "Smart Kitchen - A Measurement System" would like to declare our honest sense of gratitude to our institution - Sri Lanka Institute of Information Technology (SLIIT). We are acutely beholden to whose help, stimulating suggestions, knowledge, experience, and courage helped us in all the times of study and analysis of the project in the pre and post research period. Also very special thanks to Dr. Yasas Jayaweera for their endless support given at times of difficulty as well as to our seniors and the lecture panel. The completion of this undertaking could not have been possible without the participation and assistance of so many people whose names may not all be enumerated.

\section{REFERENCES}

[1] [1]P. Lamkin, "Connected cooking: The best smart kitchen devices and appliances", Wareable, 2017. [Online]. Available: https://www.wareable.com/smarthome/best-smart-kitchen-devices.

[2] [2]"LPG weight \& LPG leakage detection System using SMS", Microtronics Technologies, 2017. [Online]. Available: http://www.projectsof8051.com/gsm-basedlpg-weight-and-lpg-leakage-detection-system/.

[3] [3]R. Nunes and J. Delgado, "An Internet application for home automation", Electrotechnical Conference, 2000. MELECON 2000. 10th Mediterranean, 2002.

[4] [4]S. Chattoraj, "Smart Home Automation based on different sensors and Arduino as the master controller", International Journal of Scientific and Research Publications, vol. 5, no. 10, 2015.

[5] [5]S. B.M, R. C, M. S.R, P. R, P. K. Mane and K. S, "LPG Detection, Measurement and Booking System", IJRSI, vol., no., pp. 7-10, 2014.

[6] [6]A. Talgeri, A. Kumar B A and A. B, "Domotics - A Cost Effective Smart Home Automation System Using Wifi as Network Infrastructure", Int. Journal of Engineering Research and Applications, vol. 4, no. 8, pp. 52-55, 2014.

[7] [7]R. Kamoda, M. Ueda, T. Funatomi, M. Iiyama and M. Minoh, "Grocery Re-identification using Load Balance Feature on the Shelf for Monitoring Grocery Inventory."

[8] [8]F. M.L.K.E., F. W.D.P., A. G.R.S.T., J. N. and G. I., "Smart Kitchen Mate A Home Budget Management System for Food Items."

[9] [9]"Cite a Website - Cite This For Me", Lifehacker.com, $2017 . \quad$ [Online]. Available: https://lifehacker.com/5989558/top-10-ways-you-canwaste-money-without-realizing-it.

[10] [10]"Wireless Home Automation using IOT (Internet Of Things)", ElProCus - Electronic Projects for Engineering Students, 2017. [Online]. Available: https://www.elprocus.com/wireless-home-automationusing-internet-of-things/. 